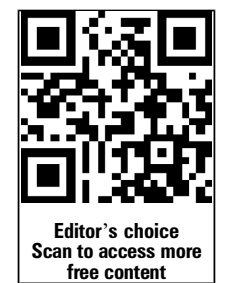

${ }^{1}$ FIFA, Zürich, Switzerland

${ }^{2}$ Schulthess Clinic, Lengghalde

2, 8008 Zurich, Switzerland

${ }^{3}$ Lausanne Anti-Doping

Laboratory, Centre Hospitalier

Universitaire Vaudois et

Université de Lausanne,

Lausanne, Switzerland

${ }^{4}$ The Brighton Centre for

Regenerative Medicine (BCRM)

University of Brighton,

Hillbrow, Eastbourne, UK

\section{Correspondence to}

Dr Jiri Dvorak, FIFA,

FIFA-Strasse 20

Zürich CH-8044, Switzerland; jiri.dvorak@f-marc.com

Accepted 24 February 2014

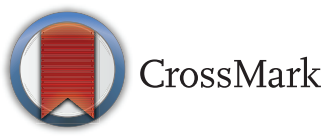

To cite: Dvorak J, Saugy M, Pitsiladis YP. Br J Sports

Med 2014;48:807-809.

\title{
Challenges and threats to implementing the fight against doping in sport
}

\author{
Jiri Dvorak, ${ }^{1,2}$ Martial Saugy, ${ }^{3}$ Yannis P Pitsiladis ${ }^{4}$
}

\section{ABSTRACT}

Prominent doping cases in certain sports have recently raised public awareness of doping and reinforced the perception that doping is widespread. Efforts to deal with doping in sport have intensified in recent years, yet the general public believes that the 'cheaters' are ahead of the testers. Therefore, there is an urgent need to change the antidoping strategy. For example, the increase in the number of individual drug tests conducted between 2005 and 2012 was approximately 90000 and equivalent to an increase of about $50 \%$, yet the number of adverse analytical findings remained broadly the same. There is also a strikingly different prevalence of doping substances and methods in sports such as a $0.03 \%$ prevalence of anabolic steroids in football compared to $0.4 \%$ in the overall WADA statistics. Future efforts in the fight against doping should therefore be more heavily based on preventative strategies such as education and on the analysis of data and forensic intelligence and also on the experiences of relevant stakeholders such as the national antidoping organisations, the laboratories, athletes or team physicians and related biomedical support staff. This strategy is essential to instigate the change needed to more effectively fight doping in sport.

\footnotetext{
"We can't solve problems by using the same kind of thinking we used when we created them." Albert Einstein
}

\section{A HISTORICAL PERSPECTIVE}

Drug testing of athletes was first introduced at the FIFA World Cup of 1966 in England and at the Olympic Games of 1968 in Mexico City, instigated by the deaths of athletes participating in the Rome Olympic Games of 1960 and the Tour de France in 1967 linked to amphetamine and nicotinyl tartrate. This early antidoping activity culminated in the creation of the 1966 FIFA antidoping regulations that comprised a list of seven groups of prohibited substances, including narcotics and stimulants. The fundamental aims of the current antidoping policy were developed in the late 1960s by the IOC and the IFs. They are to: (1) uphold and preserve the ethics of sport, (2) safeguard the physical health and mental integrity of players and (3) ensure that all competitors have an equal chance. Since then, doping controls have been performed at most of the major sporting competitions such as the Olympic Games, World Championships in track and field and other sports, the FIFA World Cup and major cycling competitions. In the process, the IOC in the first instance, and subsequently the WADA accredited laboratories, gained considerable expertise and experience in detecting prohibited substances.

In the mid and late 1990s, nutritional supplements gained immense popularity among professional as well as also recreational athletes, and an alarming number of positive doping cases for drugs such as nandrolone were reported in different sports including football. Athletes attributed their positive steroid cases to the intake of nutritional supplements contaminated with nandrolone or other anabolic steroids. A careful examination of more than 600 nutritional supplements by the WADA accredited laboratory in Cologne ${ }^{1}$ supported this claim as $15 \%$ of the samples analysed contained anabolic androgenic steroids not reported on the label. Of great concern was the finding that the majority of contaminated nutritional supplements were freely available from fitness clubs, health-food stores and the Internet. Following the publication of these results, the IFs, led by the IOC and FIFA, launched an educational campaign warning athletes to avoid nutritional supplements that were not approved by relevant national regulatory bodies. This message was reinforced by a consensus statement ${ }^{2}{ }^{3}$ that reaffirmed the view that there was no evidence of ergogenic effects of dietary supplements (ie, a positive effect on health or performance) and strongly discouraged the indiscriminate use of any nutritional supplements. It was recommended that nutritional supplements should only be taken if advised by qualified sports nutrition professionals. ${ }^{3}$

Towards the late 1990s and early in the new century, an increasing number of athletes from a variety of different sports were being sanctioned for the use of testosterone, nandrolone and other anabolic steroids. Contaminated supplements and endogenous production as revealed in a $13 \mathrm{C}$ nandrolone excretion study in trained athletes ${ }^{4}$ may, in part at least, have impacted on these positive cases. Further support for this idea emerged from a study that found traces of the metabolites of nandrolone following $90 \mathrm{~min}$ of football in healthy volunteers. ${ }^{5}$ This finding led to a $2 \mathrm{ng}$ per $\mathrm{mL}$ cut-off level for nandrolone metabolites being introduced as an adverse analytical finding. The case of nutritional supplements illustrates the importance of research in preventing inadvertent doping.

The early efforts to systematically orchestrate the fight against doping culminated in WADA being established in Lausanne, Switzerland in 1999. WADA was created as an independent body to harmonise the fight against doping in different sports, coordinate antidoping activities and create a clearing-house for the management of positive cases. The creation of WADA has undoubtedly 
intensified the fight against doping; however, a significant proportion of athletes who deliberately cheat and are supported by their medical and paramedical personnel have managed to avoid detection and use illicit means to enhance their performance.

The introduction of recombinant human erythropoietin (rHuEPO) in the early 1990s sparked a new capacity in performance enhancement primarily for endurance sports such as cycling and track and field-detection during this time was unlikely. To help athletes avoid detection, individual physicians in countries such as Italy and Spain 'masterminded' drug prescription. This resulted in high profile cases in the Tour de France and Giro d'Italia such as Marco Pantani, Tyler Hamilton and, most recently, Lance Armstrong. Arguably, these cases represent only the 'tip of the iceberg' and exposed the insufficiencies of the current antidoping system.

During the 1990s, 'blood doping' in the form of rHuEPO use and/or blood transfusions became widespread. In response, the IFs such as the International Cycling Union (UCI), FIFA and the IOC introduced the direct urine test and the sampling of blood to detect blood manipulation as evidenced by direct and indirect parameters. The analysis of indirect blood parameters conducted during the Tour de France in 1997 revealed significantly higher levels of haemoglobin, haematocrit and reticulocytes compared to the normal population, ${ }^{67}$ while a similar analysis of blood samples from players participating in the 2002 FIFA World Cup in Korea/Japan was consistent with normative data (M Saugy, personal communication, 2002). In 2004, UCI introduced a blood test for the detection of homologous blood transfusion (M Saugy, personal communication, 2006) to enhance the analysis of indirect haematological parameters indicating blood manipulation. The two observations from the UCI and FIFA clearly indicated that the risks of blood doping abuse are different in individual endurance sports compared with Olympic team sports, for example. Consequently, appropriate risk management must be based on data from adverse or atypical analytical findings as well as after considering the estimated risk of doping in different sports. We acknowledge that the latter may be difficult to determine accurately.

\section{DOPING CONTROLS, POSITIVE CASES AND ECONOMIC COSTS}

Since WADA was established, the number of doping controls performed has constantly increased both during competition and also out of competition; this increase in testing has not prevented an unknown proportion of athletes taking performance-enhancing drugs prior to and/or during major competitions. Banned and harmful substances being available over the counter without prescription compound the situation further. Drugs such as nandrolone, stanozolol, rHuEPO, testosterone and cortisol can be purchased without difficulty over the Internet or accessed in most fitness centres worldwide. Importantly, the issue of drugs in sport is not confined to professional athletes but is increasingly becoming a problem among recreational athletes. Recreational athletes are being encouraged to increase muscle mass and strength for aesthetic reasons through readily available anabolic steroids. This practice has detrimental effects on health.

Since 2005, WADA's annual reports on global doping controls in Olympic and non-Olympic sports describe the total number of adverse analytical findings, atypical findings and the breakdown of adverse analytical findings in relation to different prohibited substances. From these statistics, IFs can compare their respective prevalence with the data presented by WADA and evaluate doping trends. As mentioned earlier, the increase in the number of individual drug tests conducted between 2005 and 2012 is approximately 90000 , an increase of about $50 \%$, yet the number of adverse analytical findings has remained broadly the same ${ }^{8}$ (table 1). Similarly, the reported adverse analytical findings for anabolic steroids between 2005 and 2012 range from 715 in 2007 to 1038 in $2012^{8}$ - a modest increase. The implications of these important statistics are that increasing the number of doping tests will not necessarily result in a corresponding increase in the number of adverse analytical findings.

The annual WADA statistics on global doping controls have also allowed the FIFA Anti-Doping Unit to establish a detailed account of the adverse analytical findings and true positive doping cases (table 2). As reflected in all other sports, there was a substantial increase in drug testing between 2005 and 2012, yet the number of true positive sanctioned cases remained constant. ${ }^{8}$ A more detailed examination of these results reveals that about $50 \%$ of positive cases can be attributed to cannabinoids, and the remaining to other substances such as cocaine, stimulants and diuretics. Notably, the number of positive cases for the abuse of anabolic steroids and hormones remains, on average, at $0.03 \%$ with the exception of 2011, when five North Korean women tested positive for anabolic steroids during the FIFA Women's World Cup. ${ }^{9}$ Furthermore, the prevalence of doping substances and methods in sports is strikingly different. For example, the prevalence of anabolic steroids in football is $0.03 \%$ compared to $0.4 \%$ in the overall WADA statistics (table 1 ), and there has not been a single case of rHuEPO in football during these 8 years. While this may be a reflection of imperfect testing, this does not impact on any comparison between adverse analytical findings and positive doping cases between IFs. These prevalence data serve to reinforce the need for 'more intelligent testing'.

Large financial resources are needed to implement the current antidoping policy. The cost of organising, conducting, analysing and managing a single doping test is estimated at approximately US $\$ 1000$ on average. Consequently, given the number of doping tests conducted on average each year globally, the current estimate for the annual cost for the fight against doping

Table 1 Statistical data provided by WADA

\begin{tabular}{|c|c|c|c|}
\hline WADA statistics & $\begin{array}{l}\text { Total AAF } \\
\text { Without ATF }\end{array}$ & $\begin{array}{l}\text { Anabolic steroids } \\
\text { Without } T / E \text { ratio }\end{array}$ & $\begin{array}{l}\text { Anabolic steroids } \\
\text { Football }\end{array}$ \\
\hline $\begin{array}{l}2012 \\
267654\end{array}$ & $3190(1.2 \%)$ & $1038(0.38 \%)$ & $11(0.04 \%)$ \\
\hline $\begin{array}{l}2011 \\
243193\end{array}$ & 2885 (1.19\%) & 1191 (0.49\%) & $18(0.06 \%)$ \\
\hline $\begin{array}{l}2010 \\
258267\end{array}$ & $2790(1.08 \%)$ & $1243(0.48 \%)$ & $10(0.05 \%)$ \\
\hline $\begin{array}{l}2009 \\
277928\end{array}$ & $3091(1.1 \%)$ & $978(0.43 \%)$ & $5(0.02 \%)$ \\
\hline $\begin{array}{l}2008 \\
274615\end{array}$ & $2956(1.08 \%)$ & $1093(0.39 \%)$ & $9(0.03 \%)$ \\
\hline $\begin{array}{l}2007 \\
223898\end{array}$ & 4402 (1.97\%) & $715(0.31 \%)$ & $15(0.05 \%)$ \\
\hline $\begin{array}{l}2006 \\
198143\end{array}$ & 3887 (1.96\%) & $842(0.42 \%)$ & $10(0.04 \%)$ \\
\hline $\begin{array}{l}2005 \\
183337\end{array}$ & 3909 (2.13\%) & $732(0.40 \%)$ & $11(0.05 \%)$ \\
\hline $\begin{array}{l}\text { Total } \\
1927035\end{array}$ & $27110(1.4 \%)$ & $7832(0.4 \%)$ & $89(0.04 \%)$ \\
\hline
\end{tabular}


Table 2 Statistical data provided by FIFA, indicating true positive cases, meaning sanctioned by the FIFA disciplinary committee and reported to WADA

\begin{tabular}{|c|c|c|c|c|c|c|c|c|c|}
\hline & 2005 & 2006 & 2007 & 2008 & 2009 & 2010 & 2011 & 2012 & Total \\
\hline Total samples analysed & 23478 & 25727 & 28313 & 33445 & 32526 & 30398 & 28587 & 28008 & 230482 \\
\hline Positive cases total & $76(0.32 \%)$ & $89(0.35 \%)$ & $103(0.36 \%)$ & $82(0.36 \%)$ & $72(0.22 \%)$ & $105(0.35 \%)$ & $119(0.42 \%)$ & $106(0.38 \%)$ & $752(0.33 \%)$ \\
\hline Cannabinoids & $36(0.15 \%)$ & $52(0.2 \%)$ & $38(0.13 \%)$ & $40(0.12 \%)$ & $30(0.1 \%)$ & $42(0.14 \%)$ & $40(0.14 \%)$ & $40(0.14 \%)$ & $318(0.14 \%)$ \\
\hline Anabolic steroids and hormones & $11(0.05 \%)$ & $10(0.04 \%)$ & $15(0.05 \%)$ & $9(0.03 \%)$ & $5(0.02 \%)$ & $14(0.05 \%)$ & $18(0.06 \%)$ & $11(0.04 \%)$ & $93(0.04 \%)$ \\
\hline Others (eg, cocaine, stimulants, diuretics) & $29(1.12 \%)$ & $27(0.10 \%)$ & $50(0.18 \%)$ & $33(0.1 \%)$ & $37(0.11 \%)$ & $49(0.16 \%)$ & $61(0.21 \%)$ & $55(0.2 \%)$ & $341(0.15 \%)$ \\
\hline
\end{tabular}

is approximately US\$300 million. In football (soccer), this cost amounts to approximately US\$30 million per year. Based on these statistics, it costs around US\$3 million to catch one cheat in football for anabolic steroids. Similarly, catching a cheat for any other prohibited substance, including marijuana, could cost up to US\$300 000. While this analysis is clearly overly simplistic, it serves to highlight the need for considered risk assessments that take into account the specifics of the sport (ie, individual or team) as well as the standard of the athletes (ie, professional, semiprofessional, amateur) as these factors significantly influence the likelihood of doping. Thus, there is need for a comparative study of the prevalence of adverse analytical findings and positive cases among different sports with particular emphasis on high-profile competitions such as World Cups, Olympic Games, World Championships and other major competitions that attract the attention of the public and substantial sponsorship (eg, Tour de France, Giro d'Italia, Super Bowl). A similar approach was recently recommended by the Australian Sports Commission following clear evidence of systematic doping in Australian Rule Football. ${ }^{10}$ This notion is further reinforced by the 2015 World Anti-Doping Code that specifies the need to consider sport-specific doping patterns when implementing effective prevention.

\section{A FUTURE PERSPECTIVE}

The fight against doping in sport can be considered analogous to major public health challenges. ${ }^{11}$ For instance, testing campaigns are established to determine the prevalence of a particular disease ('surveillance'). Once the prevalence has been correctly determined, targeted treatment campaigns follow. The rationale for such an approach is, first, to screen relevant biomarker(s) of disease and then to monitor their prevalence in a targeted population using appropriate diagnostic tools. As such, populations at risk and the tools employed are quite different depending on the disease.

Although current antidoping tools are universal and applied equally to all athletes, athletes who wish to dope typically adopt strategies that depend on the type of sport. ${ }^{11}$ For example, within individual sports, endurance athletes would choose different substances and methods to illicitly improve their performance than would athletes who depend primarily on strength and power. The situation in team sports is likely to be different as results depend primarily on the collective team performance. It is essential, therefore, to analyse the prevalence and characteristics of doping in different sports, define the population at risk and introduce targeted in and out of competition testing.

Advances in the biological profiling of athletes in a longitudinal manner as per the Athlete Biological Passport (ABP), which allows results from in and out of competition to be explored, are promising but costly. For example, the molecular signature of rHuEPO doping has been discovered recently. This success provides the strongest evidence until now that state-of-the-art 'omics' technologies have the potential to significantly strengthen the current ABP approach and contribute to other traditional antidoping tests. ${ }^{12}$ A next generation ABP that also combines blood and steroid profiles is expected to be a substantial improvement. Such an approach will better identify a range of doping substances, including recombinant human growth hormone, and provide authorities with well substantiated evidence of the occurrence of blood transfusions. Consequently, a more effective ABP will have a stronger deterrent effect and substantially reduce costs in the long term, by requiring fewer antidoping tests.

A number of different approaches, some of which are summarised in this May 2014 issue of $B J S M,{ }^{10}$ have been proposed to more intelligently target testing. These ideas have emerged following careful consideration of the current body of knowledge and the extensive antidoping experience of IFs such as UCI, the IAAF and FIFA. Despite this wealth of knowledge, there is an urgent need to conduct more studies aimed at generalising successful approaches and results to create strong evidence-based justification to change the strategy in the fight against doping.

In addition to tried and tested approaches, a better strategy will require new approaches developed from 'out-of-the-box' thinking. Antidoping organisations and IFs could also consider further enhancing the involvement of sports physicians in the fight against doping. Sports physicians are required by law to abide by professional and ethical rules. ${ }^{13}$ As such, they could be asked to include the results of all regularly performed blood and urine tests (eg, periodic health examination, precompetition medical assessment) for biological profiling (ABP). ${ }^{14}{ }^{15}$ In addition to being an important contribution, such an approach would also reduce costs and is in line with emerging evidence to support its feasible and reliability. ${ }^{16}$

\section{CONCLUSION}

A sustainable and successful fight against doping in sport will depend largely on establishing close partnerships between medical and related biomedical support staff within IFs, the national antidoping organisations, athlete/team physicians and the expert laboratories. These partnerships will ensure successful implementation of the 2015 World Anti-Doping Code that focuses on prevention through education across the different age groups and different sports as well as the application of an enhanced ABP that acts as a significant deterrent while at the same time encouraging more intelligent testing. This overall strategy is essential to instigate the change needed to more effectively fight doping in sport.

Contributors JD, MS and YPP contributed to the concept, development and writing of this article.

Competing interests None.

Provenance and peer review Not commissioned; internally peer reviewed. 


\section{REFERENCES}

1 Geyer H, Parr MK, Mareck U, et al. Analysis of non-hormonal nutritional supplements for anabolic-androgenic steroids - results of an international study. Int J Sports Med 2004;25:124-9.

2 FIFA/F-MARC. Nutrition for football: the FIFA/F-MARC Consensus Conference. J Sports Sci 2006;24:663-4.

3 Hespel P, Maughan RJ, Greenhaff PL. Dietary supplements for football. J Sports Sci 2006;24:749-61.

4 Baume N, Avois L, Schweizer C, et al. [13C]Nandrolone excretion in trained athletes: interindividual variability in metabolism. Clin Chem 2004;50:355-64.

5 Baume N, Avois L, Sottas PE, et al. Effects of high-intensity exercises on 13C-nandrolone excretion in trained athletes. Clin J Sport Med 2005;15: 158-66.

6 Robinson N, Schattenberg L, Zorzoli M, et al. Haematological analysis conducted at the departure of the Tour de France 2001. Int J Sports Med 2005;26:200-7.

7 Saugy M, Robinson N, Saudan C, et al. Human growth hormone doping in sport. Br J Sports Med 2006;40(Suppl 1):i35-9.

8 WADA Testing Figures. 2013. http://www.wada-ama.org/Documents/Resources/ Testing-Figures [cited 2014].

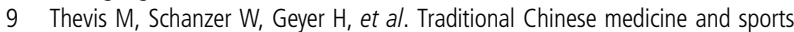
drug testing: identification of natural steroid administration in doping control urine samples resulting from musk (pod) extracts. Br J Sports Med 2013;47:109-14.

10 Harcourt PR, Marclay F, Clothier B. A forensic perspective of the AFL investigation into peptides: an anti-doping investigation case study. Br J Sports Med 2014:48:810-3.

11 Saugy M, Robinson N, Grimm K, et al. Future of the fight against doping: risk assessment, biological profiling and intelligence testing. Forensic Sci Int 2011;213:1-2.

12 Pitsilasis YP, Durussel J, Rabin O. An integrative 'Omics' solution to the detection of recombinant human erythropoietin and blood doping. $\mathrm{Br} J$ Sports Med 2014;48:856-61

13 Dvorak J. Give Hippocrates a jersey: promoting health through football/sport. Br J Sports Med 2009;43:317-22.

14 Dvorak J, Grimm K, Schmied C, et al. Development and implementation of a standardized precompetition medical assessment of international elite football players-2006 FIFA World Cup Germany. Clin J Sport Med 2009;19:316-21.

15 Dvorak J, Grimm K, Schmied C, et al. Feasibility of precompetition medical assessment at FIFA World Cups for female youth players. Br J Sports Med 2012:46:1132-3.

16 Meyer T, Meister S. Routine blood parameters in elite soccer players. Int I Sports Med 2011:32:875-81. 